\title{
Second and Third Virial Coefficients for Polyisobutylene in the Vicinity of the Theta Point
}

\author{
Kazutomo AKasaka, Yo Nakamura, Takashi Norisuye, \\ and AKIO TERAMOTO \\ Department of Macromolecular Science, Osaka University, \\ Toyonaka, Osaka 560, Japan
}

(Received September 9, 1993)

\begin{abstract}
The second virial coefficient $A_{2}$ and the third virial coefficient $A_{3}$ have been determined for five polyisobutylene fractions ranging in weight-average molecular weight $M_{w}$ from $8 \times 10^{4}$ to $1.6 \times 10^{6}$ in isoamyl isovalerate at different temperatures $T$ between 21 and $37^{\circ} \mathrm{C}$ by light scattering. The theta temperature $\Theta$ where $A_{2}$ vanishes is found to be $27^{\circ} \mathrm{C}$ independent of $M_{w}$. The curve of $A_{3} v s$. $T$ obtained for each fraction has a broad minimum around $\Theta$, and the minimum becomes very shallow as $M_{w}$ decreases. These features are very similar to those observed previously for polystyrene in cyclohexane. The values of $A_{3}$ at $\Theta$ are in the range between $3 \times 10^{-4}$ and $7 \times 10^{-4} \mathrm{~mol} \mathrm{~g}^{-3} \mathrm{~cm}^{6}$, and thus demonstrate the breakdown of the binary cluster approximation to $A_{3}$ for polyisobutylene near $\Theta$. It is shown that existing theories of $A_{2}$ and $A_{3}$ are incapable of explaining consistently the positive $A_{3}$ at $\Theta$ and the molecular weight independence of $\Theta$ in the range of $M_{w}$ studied, as found to be the case for polystyrene.

KEY WORDS Second Virial Coefficient / Third Virial Coefficient / Polyisobutylene / Theta Point / Three-Segment Interaction / Two-Parameter Theory / Light Scattering /
\end{abstract}

In previous work, ${ }^{1}$ we found from light scattering measurements that the third virial coefficient $A_{3}$ for polystyrene in cyclohexane remains positive at the theta temperature $\Theta$ where the second virial coefficient $A_{2}$ equals zero. This reveals the breakdown of the two-parameter theory ${ }^{2}$ for $A_{3}$ near the theta point, since the theory predicts that $A_{2}$ and $A_{3}$ simultaneously vanish at $\Theta$. Another important finding in the previous study was that $A_{3}$ increases with a decrease in temperature below $\Theta$. This is also contrasted to the twoparameter theory prediction that $A_{3}$ should become negative below $\Theta$.

It is intriguing to see whether the above findings are common to other linear flexible chains in theta solvents. This motivated us to perform similar light scattering measurements on sharp fractions of polyisobutylene (PIB), another typical flexible polymer. Isoamyl isovalerate (IAIV) was chosen as the solvent, because it brings the polymer to the theta state at room temperature and gives a refractive index increment large enough for light scattering experiment. ${ }^{3,4}$ Data were obtained not only for $A_{2}$ and $A_{3}$ but also for the $z$ average mean-square radius of gyration $\left\langle S^{2}\right\rangle_{z}$ as functions of molecular weight and temperature. The virial coefficient data at $\Theta$ are discussed below in relation to three-segment interactions.

\section{EXPERIMENTAL}

\section{Preparation of Solutions}

Five PIB fractions with molecular weights of $8 \times 10^{4}-1.6 \times 10^{6}$ (in cyclohexane) were chosen from the previously investigated samples. ${ }^{5}$ The ratios of the weight-average to number-average molecular weight estimated from gel permeation chromatography (GPC) in chloroform were in the range $1.08-1.10 .^{5}$ 
The solvent IAIV (Tokyo Kasei Kogyo Co.) was washed twice with $2 N$ aqueous sodium carbonate and three times with water, dehydrated with anhydrous potassium carbonate, and fractionally distilled twice under reduced nitrogen atmosphere. Each polymer sample was mixed with the solvent in a dry bag filled with nitrogen. The mixture was gently stirred in the dark for $2-10$ days at $35^{\circ} \mathrm{C}$, with care taken to ensure complete dissolution of the polymer and to prevent it from degradation.

The polymer mass concentration $c$ of each solution was calculated from the polymer weight fraction with the solution density $\rho$. The density was measured at 20,30 , and $40^{\circ} \mathrm{C}$ using a bicapillary pycnometer of $30 \mathrm{~cm}^{3}$ capacity. The results for $c \precsim 3 \times 10^{-2} \mathrm{~g} \mathrm{~cm}^{-3}$ were found to be represented by

$$
\begin{array}{ll}
\rho=0.85503+0.0714 c & \left(20^{\circ} \mathrm{C}\right) \\
\rho=0.84631+0.0761 c & \left(30^{\circ} \mathrm{C}\right) \\
\rho=0.83762+0.0806 c & \left(40^{\circ} \mathrm{C}\right)
\end{array}
$$

where both $c$ and $\rho$ are expressed in units of $\mathrm{g} \mathrm{cm}^{-3}$. Necessary values of $\rho$ at different temperatures were obtained by interpolation.

\section{Light Scattering Measurement}

Light scattering measurements were carried out at temperatures ranging from 21 to $37^{\circ} \mathrm{C}$ on a Fica-50 light scattering photometer with vertically polarized incident light of $436 \mathrm{~nm}$ wavelength in an angular range from 30 to $150^{\circ}$. Pure benzene at $25^{\circ} \mathrm{C}$ was used to calibrate the apparatus. Its Rayleigh ratio at $436 \mathrm{~nm}$ was taken to be $46.5 \times 10^{-6} \mathrm{~cm}^{-1}$, and its depolarization ratio was determined to be 0.40 by the method of Rubingh and $\mathrm{Yu}{ }^{7}$ The specific refractive index increment $\partial n / \partial c$ for PIB in IAIV at $436 \mathrm{~nm}$ was determined at $25,30,35$, and $40^{\circ} \mathrm{C}$ using a modified SchulzCantow type differential refractometer, with the result that for $c \precsim 4.5 \times 10^{-2} \mathrm{~g} \mathrm{~cm}^{-3}$

$$
\begin{array}{ll}
\partial n / \partial c=0.1100-0.06 c & \left(25^{\circ} \mathrm{C}\right) \\
\partial n / \partial c=0.1113-0.08 c & \left(30^{\circ} \mathrm{C}\right)
\end{array}
$$

$$
\begin{array}{ll}
\partial n / \partial c=0.1127-0.11 c & \left(35^{\circ} \mathrm{C}\right) \\
\partial n / \partial c=0.1140-0.14 c & \left(40^{\circ} \mathrm{C}\right)
\end{array}
$$

where $\partial n / \partial c$ is expressed in units of $\mathrm{cm}^{3} \mathrm{~g}^{-1}$. Necessary $\partial n / \partial c$ values at different temperatures were obtained by interpolation. The infinite-dilution value of $0.1100 \mathrm{~cm}^{3} \mathrm{~g}^{-1}$ at $25^{\circ} \mathrm{C}$ is in excellent agreement with the values 0.1091 and $0.1103 \mathrm{~cm}^{3} \mathrm{~g}^{-1}$ reported by Matsumoto et $a l .{ }^{3}$ and by Abe et al. ${ }^{4}$ respectively.

Refractive indices of IAIV were determined by à precision sugar refractometer (Bausch and Lomb Co.) to be $1.421_{7}$ at $20.1^{\circ} \mathrm{C}, 1.417_{5}$ at $29.9^{\circ} \mathrm{C}$, and $1.412_{8}$ at $39.9^{\circ} \mathrm{C}$ for light of the wavelength $436 \mathrm{~nm}$.

\section{RESULTS}

\section{Molecular Weight and Virial Coefficients}

Scattering intensity data obtained were extrapolated to zero angle using Berry's square-root plot $^{8}$ of $\left(K c / R_{\theta}\right)^{1 / 2} v s \cdot \sin ^{2}(\theta / 2)$, where $K$ is the optical constant and $R_{\theta}$, the reduced scattering intensity at a scattering angle $\theta$. Figure 1 illustrates the concentration dependence of $K c / R_{\theta}$ at zero scattering angle, i.e., $K c / R_{0}$, for $\mathrm{PIB}$ fraction $\mathrm{S}-14 \mathrm{~B}$ at the indicated temperatures $T$. The curves fitting the data points at the respective $T$ bend upward and appear to converge to a common ordinate intercept.

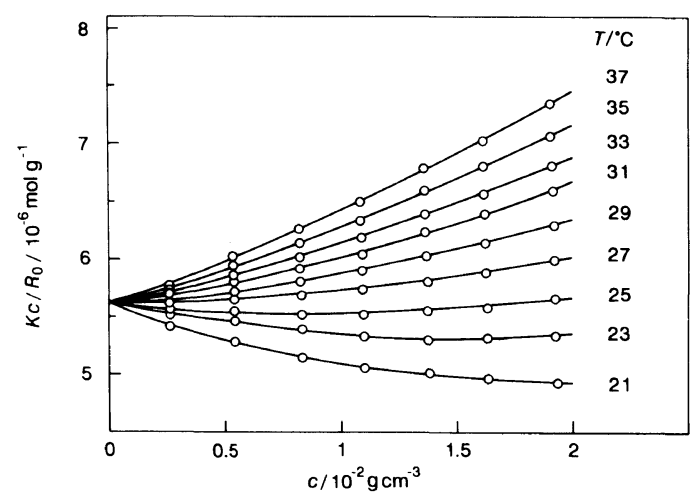

Figure 1. Concentration dependence of scattering intensity at zero angle for PIB fraction S-14B in IAIV at indicated temperatures. 


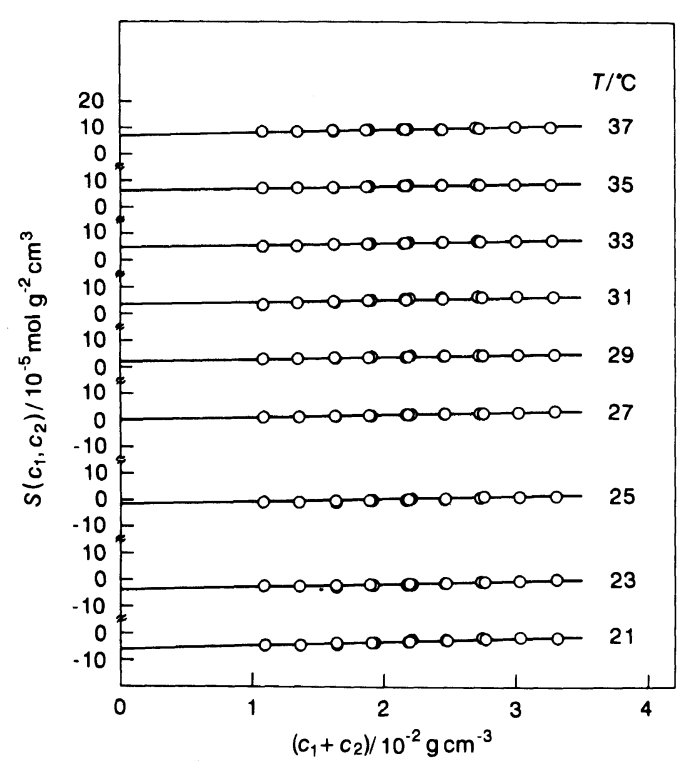

Figure 2. Bawn plots constructed from the data in Figure 1 according to eq 1 .

The values of $K c / R_{0}$ at finite concentrations were analyzed using the expression ${ }^{9,10}$ (originally derived by Bawn et al. ${ }^{11}$ for osmotic pressure)

$$
\begin{aligned}
S\left(c_{1}, c_{2}\right) & \equiv \frac{\left(K c / R_{0}\right)_{c=c_{2}}-\left(K c / R_{0}\right)_{c=c_{1}}}{c_{2}-c_{1}} \\
& =2 A_{2}+3 A_{3}\left(c_{1}+c_{2}\right)+\cdots
\end{aligned}
$$

where $c_{1}$ and $c_{2}$ denote different polymer mass concentrations. The Bawn plots of $S\left(c_{1}, c_{2}\right) v s$. $c_{1}+c_{2}$ constructed from the data in Figure 1 are shown in Figure 2. The plotted points at each $T$ follow a straight line, whose intercept and initial slope permit us to determine $A_{2}$ and $A_{3}$ separately. Similar plots for five PIB fractions at $27.0^{\circ} \mathrm{C}$ are displayed in Figure 3.

The weight-average molecular weight $M_{w}$ can be obtained by extrapolation of the apparent molecular weight defined by ${ }^{9}$

$$
M_{\mathrm{app}}=\left[K c / R_{0}-2 A_{2} c-3 A_{3} c^{2}\right]^{-1}
$$

to infinite dilution. Figure 4 shows the plots of $M_{\text {app }} v s . c$ for five fractions at $27.0^{\circ} \mathrm{C}$. The plotted points for each fraction fall essentially on a horizontal line, allowing unambiguous

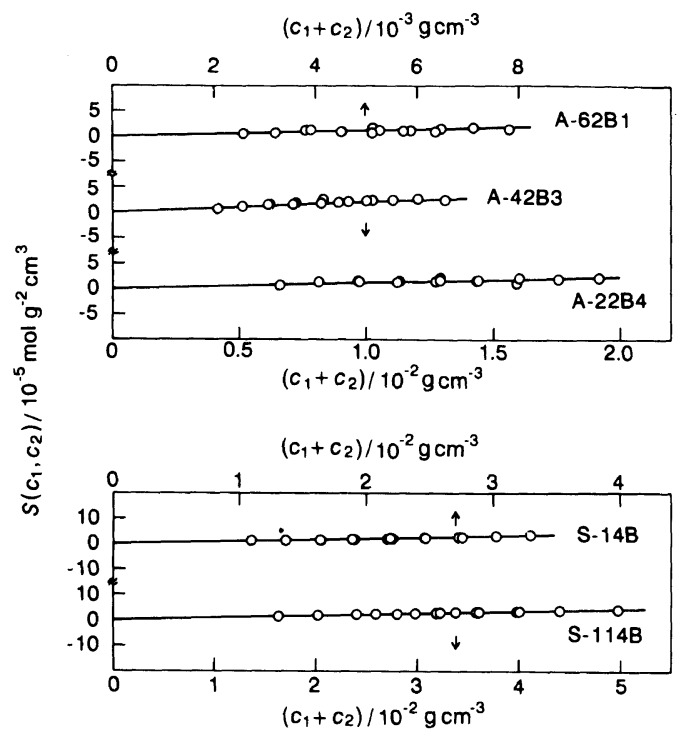

Figure 3. Bawn plots for the indicated PIB fractions in IAIV at $27^{\circ} \mathrm{C}$.

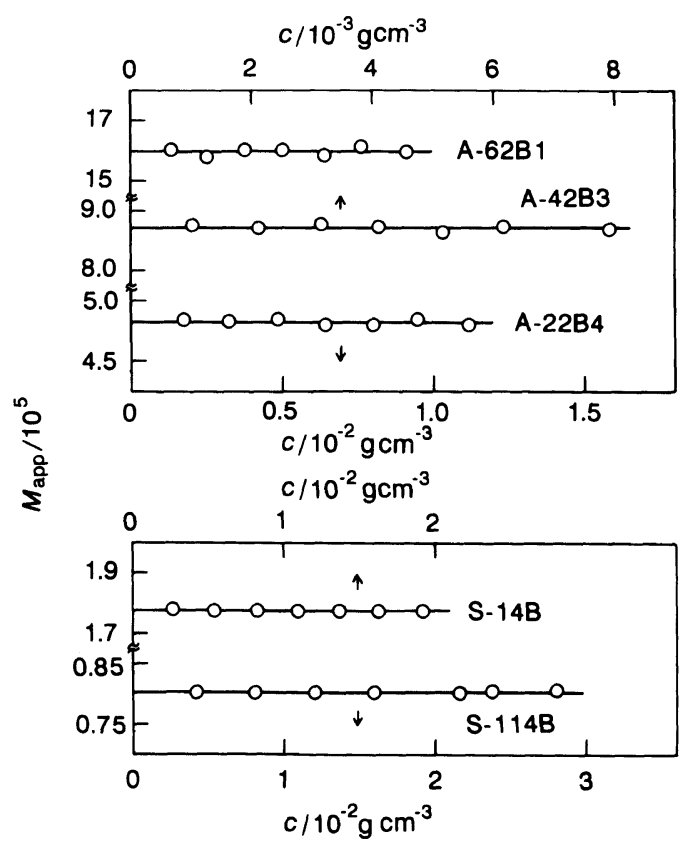

Figure 4. Plots of $M_{\text {app }} v s . c$ for the indicated PIB fractions in IAIV at $27^{\circ} \mathrm{C} . M_{\text {app }}$ is defined by eq 2 .

determination of $M_{w}$. The $M_{w}$ values determined at different temperatures agreed within $\pm 1.6 \%$ for any fractions.

The values of $A_{2}$ for five PIB fractions in 
Table I. Second virial coefficients for PIB fractions in IAIV at different temperatures

\begin{tabular}{cccccc}
\hline \multicolumn{5}{c}{$A_{2} / 10^{-5} \mathrm{~mol} \mathrm{~g}^{-2} \mathrm{~cm}^{3}$} \\
\hline$T /{ }^{\circ} \mathrm{C}$ & $\begin{array}{c}\mathrm{S}-114 \mathrm{~B} \\
\end{array}$ & $\begin{array}{c}\mathrm{S}-14 \mathrm{~B} \\
M_{w}=8.04 \times 10^{4}\end{array}$ & $\begin{array}{c}\mathrm{A}-22 \mathrm{~B} 4 \\
M_{w}=17.8 \times 10^{4}\end{array}$ & $\begin{array}{c}\mathrm{A}-42 \mathrm{~B} 3 \\
M_{w}=48.2 \times 10^{4}\end{array}$ & $\begin{array}{c}M_{w}=87.2 \times 10^{4} \\
M_{w}=160 \times 10^{4}\end{array}$ \\
\hline 21.0 & -2.3 & -3.1 & -3.0 & -3.3 & -4.0 \\
23.0 & -1.4 & -2.0 & -2.0 & -1.9 & -2.0 \\
25.0 & -0.6 & -0.9 & -1.0 & -0.8 & -1.0 \\
27.0 & 0.0 & 0.0 & 0.0 & 0.0 & 0.0 \\
29.0 & 1.0 & 1.0 & 1.0 & 0.9 & 0.8 \\
31.0 & 2.0 & 1.8 & 1.8 & 2.3 & 1.3 \\
33.0 & 3.0 & 2.5 & 2.5 & 2.8 & 1.9 \\
35.0 & 3.5 & 3.0 & 2.8 & 3.3 & 2.4 \\
37.0 & 4.0 & 3.5 & 3.5 & & 3.0 \\
\hline
\end{tabular}

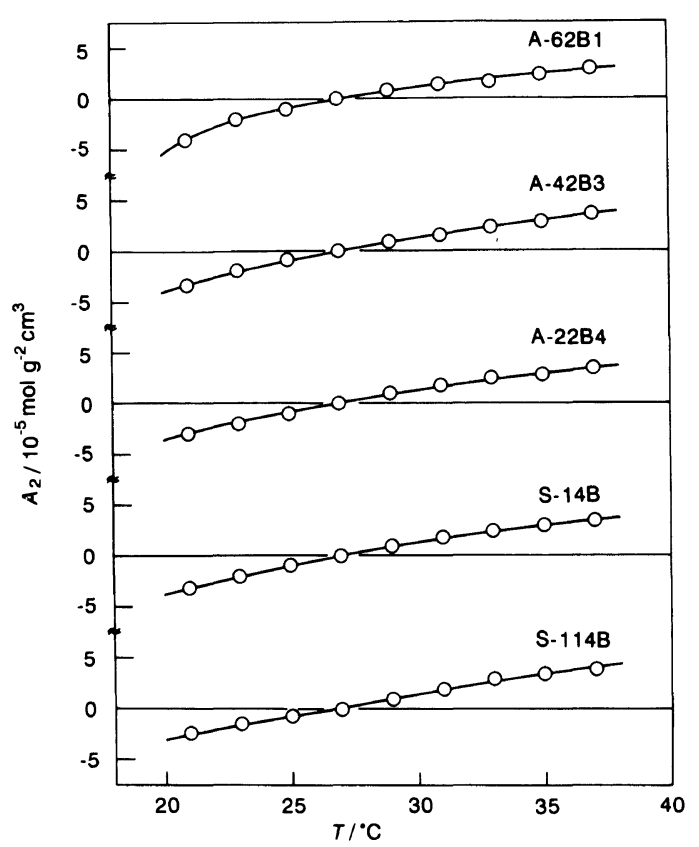

Figure 5. Temperature dependence of $\mathrm{A}_{2}$ for the indicated PIB fractions in IAIV.

IAIV at different temperatures are summarized in Table I, together with those of $M_{w}$ at $27^{\circ} \mathrm{C}$, and plotted against $T$ in Figure 5. The theta temperature where $A_{2}=0$ is determined as $27.0 \pm 0.05^{\circ} \mathrm{C}$ independent of $M_{w}$ in the molecular weight region studied. The molecularweight independence of $\Theta$ is consistent with the findings by Matsumoto et al. ${ }^{3}\left(1.6 \times 10^{5}<\right.$

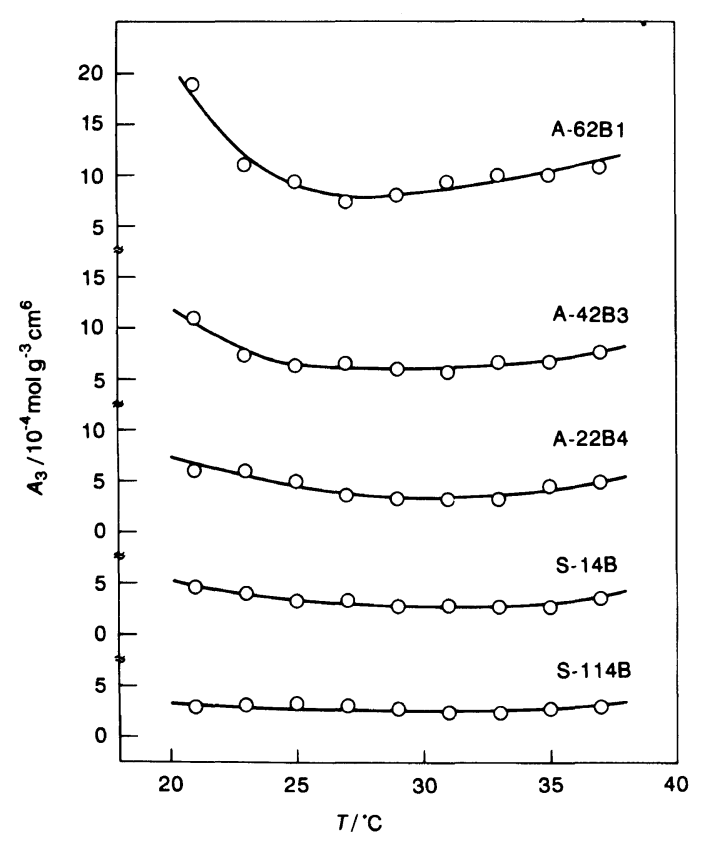

Figure 6. Temperature dependence of $\mathrm{A}_{3}$ for the indicated PIB fractions in IAIV.

$\left.M_{w}<4.7 \times 10^{6}\right)$ and by Abe et $a .^{4}(4.2 \times$ $\left.10^{5}<M_{w}<1.2 \times 10^{6}\right)$. However, our $\Theta$ value is higher by about $2-5^{\circ} \mathrm{C}$ than those determined by these previous workers. This discrepancy in $\Theta$ among the three groups may be due to some subtle difference in the purity of the IAIV used.

The temperature dependence of $A_{3}$ is shown 
Table II. Third virial coefficients for PIB fractions in IAIV at different temperatures

\begin{tabular}{cccccc}
\hline \multicolumn{5}{c}{$A_{3} / 10^{-4} \mathrm{~mol} \mathrm{~g}^{-3} \mathrm{~cm}^{6}$} \\
\hline$T /{ }^{\circ} \mathrm{C}$ & $\begin{array}{c}\mathrm{S}-114 \mathrm{~B} \\
\end{array}$ & $\begin{array}{c}\text { S-14B } \\
M_{w}=8.04 \times 10^{4}\end{array}$ & $\begin{array}{c}\text { A-22B4 } \\
M_{w}=17.8 \times 10^{4}\end{array}$ & $\begin{array}{c}M_{w}=48.2 \times 10^{4} \\
M_{w}=87.2 \times 10^{4}\end{array}$ & $M_{w}=160 \times 10^{4}$ \\
\hline 21.0 & 3.0 & 4.7 & 6.0 & 11 & 19 \\
23.0 & 3.2 & 4.0 & 6.0 & 7.3 & 11 \\
25.0 & 3.3 & 3.3 & 5.0 & 6.3 & 9.3 \\
27.0 & 3.1 & 3.3 & 3.7 & 6.7 & 7.3 \\
29.0 & 2.8 & 2.8 & 3.3 & 6.0 & 9.0 \\
31.0 & 2.5 & 2.8 & 3.3 & 5.7 & 6.3 \\
33.0 & 2.5 & 2.8 & 3.3 & 6.7 & 10 \\
35.0 & 2.9 & 2.8 & 4.7 & 7.7 & 10 \\
37.0 & 3.1 & 3.7 & 5.0 & 11 \\
\hline
\end{tabular}

in Figure 6. The curve for each fraction has a broad, positive minimum around the theta temperature $27^{\circ} \mathrm{C}$ and the minimum becomes very shallow as $M_{w}$ decreases. These features are quite similar to what was observed previously for polystyrene in cyclohexane. ${ }^{1}$ The positive $A_{3}$ at $\Theta$ and its increase with a decrease in $T$ below $\Theta$ demonstrate that the twoparameter theory does not hold for PIB in IAIV; we note again that the theory predicts $A_{3}$ to vanish at $\Theta$ and to be negative below $\Theta$. The numerical data for $A_{3}$ are presented in Table II.

In previous work, ${ }^{1}$ we showed that $A_{3}(\Theta)$, the value of $A_{3}$ at the theta temperature, can also be estimated from the intercept of a plot of $Q v s . c$ based on the relation

$$
\begin{aligned}
Q & \equiv\left[\left(K c / R_{0}\right)-\left(1 / M_{w}\right)\right] / c^{2} \\
& =3 A_{3}+4 A_{4} c+\cdots \quad\left(A_{2}=0\right)
\end{aligned}
$$

where $A_{4}$ denotes the fourth virial coefficient. This method has an advantage that $A_{3}$ is estimated separately from $A_{4}$ even when $K c / R_{0}$ contains some contribution from $A_{4}$ in the concentration range examined, though for its practical application, a very accurate value of $M_{w}$ must be known from a separate experiment. The present $M_{w}$ values for fractions A-22B4, A-42B3, and A-62B1 agreed with those $^{5}$ determined in cyclohexane (a good solvent) within $1.3 \%$. Thus, with the $M_{w}$ values in cyclohexane, we constructed plots of $Q v s . c$ for the three fractions in IAIV at $27^{\circ} \mathrm{C}$; we omitted intensity data at low $c$ where the difference between $K c / R_{0}$ and the input value of $1 / M_{w}$ was less than $1 \%$. The plots were essentially horizontal and gave $A_{3}(\Theta)$ of $5 \times 10^{-4}, 7 \times 10^{-4}$, and $6 \times 10^{-4} \mathrm{~mol} \mathrm{~g}^{-3} \mathrm{~cm}^{6}$ for fractions A-22B4, A-42B3, and A-62B1, respectively. This $c$-independent behavior of $Q$ and the substantial agreement of these values with those in Table II lend support to our previous conviction ${ }^{1,12}$ that $A_{4}$ has a negligible contribution to $K c / R_{0}$ when the Bawn plot is linear.

As can be seen from Figure 6 or Table II, the values of $A_{3}(\Theta)$ for PIB in IAIV are relatively insensitive to $M_{w}$, being $(5 \pm 2) \times 10^{-4}$ molg ${ }^{-3} \mathrm{~cm}^{6}$. Murakami et al. ${ }^{13}$ estimated $A_{3}(\Theta)$ for PIB in benzene to be $3 \times 10^{-4}$ $\mathrm{mol} \mathrm{g}^{-3} \mathrm{~cm}^{6}$ from the osmotic pressure data of Flory and Daoust. ${ }^{14}$ This value happens to be close to our $A_{3}(\Theta)$. We note that these values are one or two orders of magnitude smaller than those in the good solvent cyclohexane. ${ }^{5}$

\section{Chain Dimensions}

Since our light scattering measurements were made at concentrations considerably higher than those usually employed in dilutesolution work, no conventional procedure 


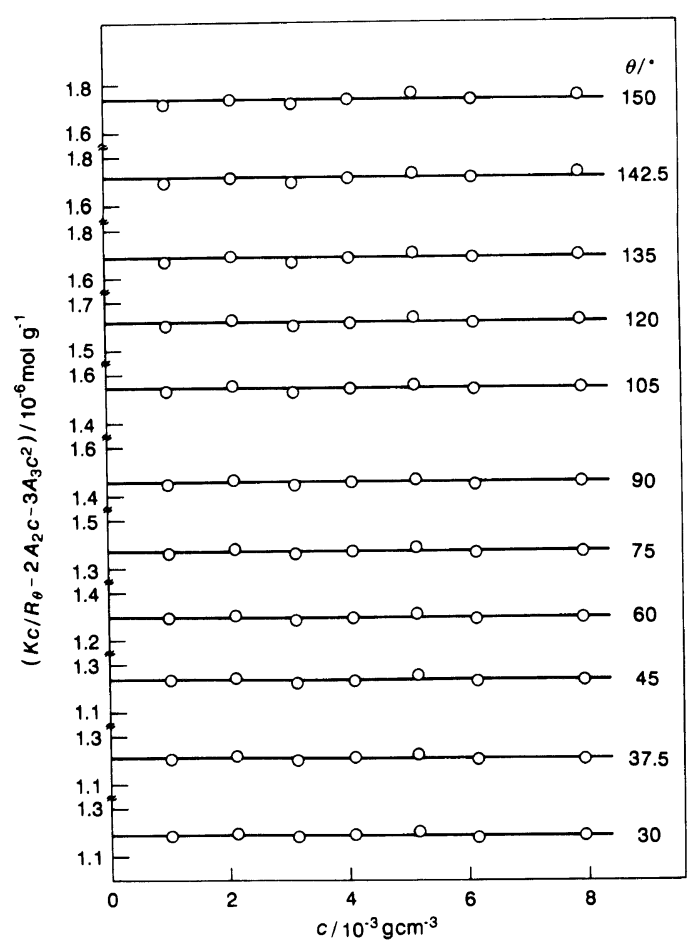

Figure 7. Concentration dependence of $\left(\mathrm{Kc} / \mathrm{R}_{\theta}-2 \mathrm{~A}_{2} \mathrm{c}-\right.$ $3 A_{3} c^{2}$ ) for PIB fraction A-42B3 in IAIV at $27^{\circ} \mathrm{C}$ and fixed scattering angles.

allowed $K c / R_{\theta}$ at fixed $\theta$ to be accurately extrapolated to infinite dilution. When the values of $\left(K c / R_{\theta}-2 A_{2} c-3 A_{3} c^{2}\right)$ calculated for fixed $\theta$ were plotted against $c$, it was found that the resulting plots for respective $\theta$ were essentially horizontal at any $T$ studied and thus gave desired values of $\left(K c / R_{\theta}\right)_{c=0}$ with high accuracy. Figure 7 illustrates such plots for fraction $\mathrm{A}-42 \mathrm{~B} 3$ at $27^{\circ} \mathrm{C}$, and the values of $\left(K c / R_{\theta}\right)_{c=0}{ }^{1 / 2}$ obtained are plotted against $\sin ^{2}(\theta / 2)$ in Figure 8, along with those for other fractions S-14B, A-22B4, and A-62B1 at $27^{\circ} \mathrm{C}$. The plotted points for each fraction follow a straight line throughout the entire range of $\theta$ examined.

The values of $\left\langle S^{2}\right\rangle_{z}{ }^{1 / 2}$ evaluated are plotted against $T$ in Figure 9, and those at the theta temperature $\left(27^{\circ} \mathrm{C}\right)$, i.e., the $\left\langle S^{2}\right\rangle_{0 z}{ }^{1 / 2}$ values, are presented in Table III, in which the $M_{w}$ data are the reproductions from Table I. The

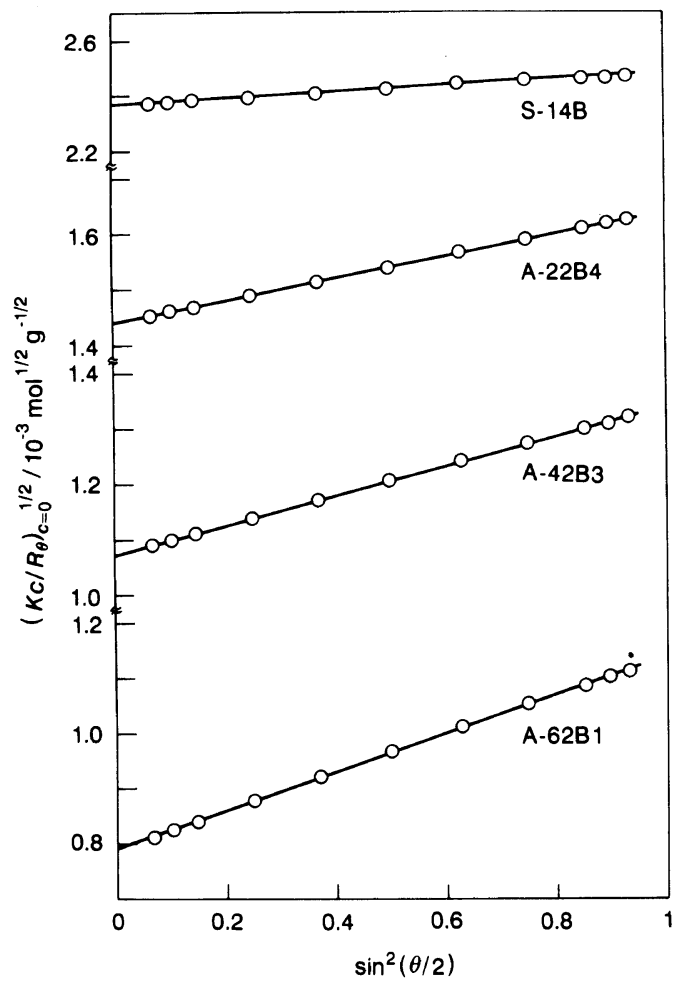

Figure 8. Plots of $\left(K c / R_{\theta}\right)_{c=0}^{1 / 2}$ vs. $\sin ^{2}(\theta / 2)$ for the indicated PIB fractions in IAIV at $27^{\circ} \mathrm{C}$.

Table III. Values of $M_{w},\left\langle S^{2}\right\rangle_{0 z}{ }^{1 / 2}$, and $[\eta]_{0}$ for PIB fractions in IAIV at the theta temperature

\begin{tabular}{|c|c|c|c|}
\hline \multirow{2}{*}{ Fraction } & \multirow{2}{*}{$M_{w} / 10^{4}$} & $\left\langle S^{2}\right\rangle_{0 z}{ }^{1 / 2}$ & {$[\eta]_{0}$} \\
\hline & & $\mathrm{nm}$ & $10^{2} \mathrm{~cm}^{3} \mathrm{~g}^{-1}$ \\
\hline S-114B & 8.04 & - & 0.304 \\
\hline S-14B & 17.8 & 13.2 & 0.472 \\
\hline A-22B4 & 48.2 & 22.1 & 0.780 \\
\hline A-42B3 & 87.2 & 29.7 & 1.05 \\
\hline A-62B1 & 160 & 39.7 & 1.42 \\
\hline
\end{tabular}

ratio of $\left\langle S^{2}\right\rangle_{0 z}$ to $M_{w}$ at $27^{\circ} \mathrm{C}$ is $(1.00 \pm 0.02) \times$ $10^{-17} \mathrm{~cm}^{2}$ independent of $M_{w}$, being consistent with the above finding from $A_{2}$ that the theta temperature is $27^{\circ} \mathrm{C}$ for PIB in IAIV. The value $1.00 \times 10^{-17} \mathrm{~cm}^{2}$ for the unperturbed dimensions $\left\langle S^{2}\right\rangle_{0 z} / M_{w}$ agrees closely with the reported data $\left(9.52 \times 10^{-18} \mathrm{~cm}^{2}\right.$ by Matsumoto et al. ${ }^{3}$ and $9.83 \times 10^{-18} \mathrm{~cm}^{2}$ by 


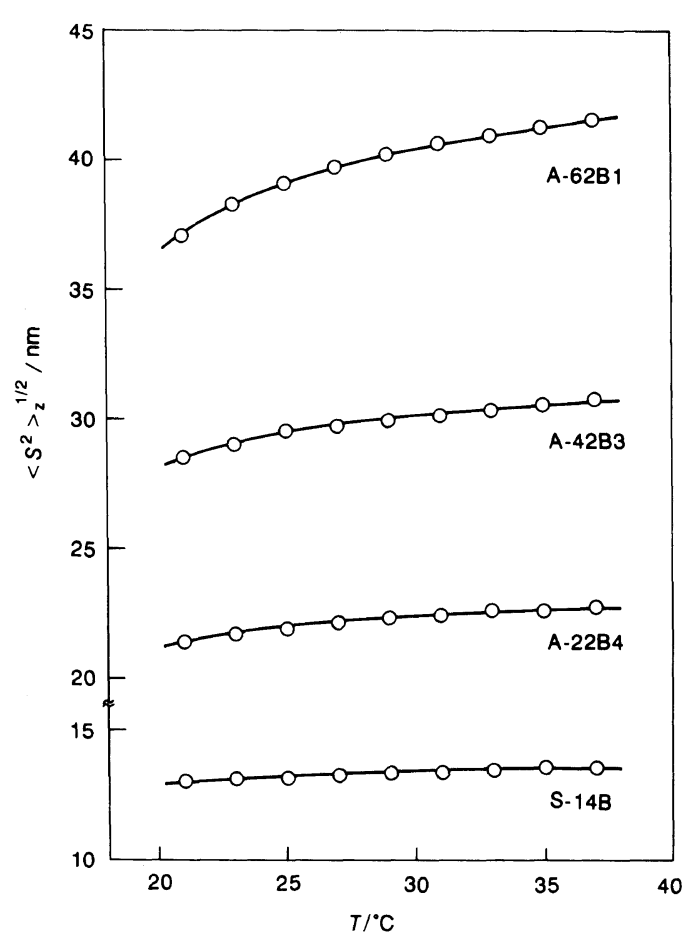

Figure 9. Temperature dependence of $\left\langle S^{2}\right\rangle_{z}{ }^{1 / 2}$ for the indicated PIB fractions in IAIV.

Konishi et al. ${ }^{15}$ ) despite the fact that the theta temperatures are at variance.

We also determined intrinsic viscosities $[\eta]_{0}$ for five PIB fractions in IAIV at $27^{\circ} \mathrm{C}$ to ascertain that the theta temperature of $27^{\circ} \mathrm{C}$ is consistent with $[\eta]_{0}$. This is substantiated by the $[\eta]$ data presented in Table III, from which it can be shown that $[\eta]_{0}$ is accurately proportional to $M_{w}{ }^{1 / 2}$. The proportionality constant $[\eta]_{0} / M_{w}{ }^{1 / 2}$ is $0.112 \mathrm{~cm}^{3} \mathrm{~g}^{-1}$. This value is again in excellent agreement with the published data $\left(0.114 \mathrm{~cm}^{3} \mathrm{~g}^{-1}\right.$ by Matsumoto et al. ${ }^{3}$ and $0.109 \mathrm{~cm}^{3} \mathrm{~g}^{-1}$ by Abe et al. ${ }^{4}$ for $\left.M_{w}>2 \times 10^{4}\right)$. In passing, our $\left\langle S^{2}\right\rangle_{0 z}$ and $[\eta]_{0}$ data yield on the average $2.4 \times 10^{23} \mathrm{~mol}^{-1}$ for the Flory viscosity factor $\Phi_{\theta}$. If correction is made for polydispersity with the $M_{z} / M_{w}$ ratio of $1.09\left(M_{z}\right.$ is the $z$-average molecular weight) from our previous GPC data ${ }^{5}$ on the assumption that the GPC curve represents the molecular weight distribution, then $\Phi_{\theta}$ for "monodisperse" PIB is obtained as $2.7 \times 10^{23}$ $\mathrm{mol}^{-1}$, a value very close to that reported by Konishi et al. ${ }^{15}$

\section{DISCUSSION}

\section{Third Virial Coefficient}

We have found that $A_{3}$ of PIB in IAIV remains positive at the theta temperature as is the case for polystyrene in cyclohexane. ${ }^{1}$ This implies that the binary cluster approximation is no longer valid for $A_{3}$ of these typical flexible polymers near the theta point. If the ternary cluster integral $\beta_{3}$ representing the interaction among three segments is incorporated into theory, $A_{3}$ near $\Theta$ is expressed by ${ }^{16,17}$

$$
A_{3}=\frac{N_{\mathrm{A}}^{2} n^{3}}{3 M^{3}}\left[\beta_{3}+O\left(\beta_{2} \beta_{3}, \beta_{3}^{2}, \beta_{2}^{3}\right)\right]
$$

or

$$
A_{3}=\frac{N_{\mathrm{A}}^{2}}{3}\left(\frac{4 \pi\left\langle S^{2}\right\rangle_{0}}{M}\right)^{3} \quad\left(z_{3}+\cdots\right)
$$

where

$$
z_{3}=\left(\frac{3}{2 \pi b^{2}}\right)^{3} \beta_{3}
$$

In these equations, $N_{\mathrm{A}}$ is the Avogadro constant, $n$ the number of segments in one chain, $\beta_{2}$ the binary cluster integral, and $b$ the segment length.

Equation 4 indicates that $A_{3}(\Theta)$ is insensitive to $M$ if both $\beta_{2}$ and $\beta_{3}$ are vanishingly small. This appears to be consistent with our $A_{3}(\Theta)$ data. Substituting $A_{3}(\Theta)=(5 \pm 2) \times 10^{-4} \mathrm{~mol}$ $\mathrm{g}^{-3} \mathrm{~cm}^{6}$ into eq 4 truncated at the leading term, we obtain $(7 \pm 3) \times 10^{-46} \mathrm{~cm}^{6}$ for $\beta_{3}$, which yields $z_{3}=0.002 \pm 0.001$ when use is made of $b=0.58 \mathrm{~nm}$ derived from our relation $\left\langle S^{2}\right\rangle_{0 z} / M_{w}=1.00 \times 10^{-17} \mathrm{~cm}^{2}$. This $z_{3}$ value is much smaller than unity, but, as discussed below, the ternary cluster interaction brings about a serious problem concerning the theta point. 


\section{Second Virial Coefficient}

If both $\beta_{2}$ and $\beta_{3}$ are taken into account to first order, it can be shown that $A_{2}$ for the Gaussian bead model is given by (see also refs 17 and 18)

$$
\begin{aligned}
A_{2}= & \frac{N_{\mathrm{A}} n^{2}}{2 M^{2}} \\
& \times\left\{\beta_{\mathrm{e}}-8\left(\frac{3}{2 \pi b^{2}}\right)^{3 / 2} \frac{\beta_{3}}{\sqrt{n}}+O\left(n^{-1}\right)\right\}
\end{aligned}
$$

where $\beta_{\mathrm{e}}$ is the effective binary cluster integral defined by

$$
\beta_{\mathrm{e}}=\beta_{2}+C \beta_{3}
$$

with

$$
C=2 \zeta\left(\frac{3}{2}\right)\left(\frac{3}{2 \pi b^{2}}\right)^{3 / 2}
$$

In eq $9, \zeta(x)$ is the Riemann zeta function of variable $x$; and $\zeta(3 / 2)(=2.612)$ comes from

$n^{-1} \sum_{i=1}^{n}(n-i) i^{-3 / 2}=\zeta\left(\frac{3}{2}\right)-4 n^{-1 / 2}+O\left(n^{-1}\right)$

for large $n$ (see ref 18 for the formulation of the ternary cluster term in $A_{2}$ ). Replacement of the sum by an integral with a cutoff parameter $\sigma$ gives $C=4 \sigma^{-1 / 2}\left(3 / 2 \pi b^{\dot{2}}\right)^{3 / 2}$ but does not alter the second term in eq $7 .^{1}$ This term is not affected by chain stiffness either if the chain is sufficiently long so that its $\left\langle S^{2}\right\rangle_{0}$ is proportional to $n$. With regard to effects of chain stiffness on $A_{2}$ near $\Theta$, the following remarks may be in order.

The Kratky-Porod wormlike chain ${ }^{19}$ mimics the PIB chain in the unperturbed state. ${ }^{4}$ When use is made of Yamakawa and Stockmayer's ring closure probability ${ }^{20}$ for the wormlike chain bead model, $C$ and the second term in eq 7 are replaced by $3.159\left(\lambda b^{\prime}\right)^{2}\left(3 / 2 \pi b^{\prime 2}\right)^{3 / 2}$ and $-8\left(\lambda b^{\prime}\right)^{3 / 2}\left(3 / 2 \pi b^{\prime 2}\right)^{3 / 2} \beta_{3} / n^{1 / 2}$, respectively (in our previous paper, ${ }^{1} \lambda b^{\prime}$ is set equal to unity). Here, $b^{\prime}$ is the bead spacing to be evaluated from $b^{\prime}=M_{0} / M_{\mathrm{L}}$ with $M_{0}$ and $M_{\mathrm{L}}$ being the molar mass of a monomer and the molar mass per unit contour length, respectively; the total contour length of the chain equals $n b^{\prime}$. Because of the presence of $\lambda^{2}, C$ for the wormlike chain (or more generally for the helical wormlike chain) ${ }^{21}$ is much smaller than that (eq 9) for the Gaussian model. On the other hand, the second term in eq 7 remains the same for long wormlike chains ${ }^{1}$ whose $\left\langle S^{2}\right\rangle_{0}$ is proportional to $n$, since $b^{\prime} \lambda^{-1}$ can be replaced by $b^{2}$ in this case. Thus, eq 7 (truncated at the second term) for the Gaussian model appears to hold for flexible chains provided that $C$ is replaced by an appropriate expression depending on the chain model relevant to the actual polymer considered.

Equation 7 indicates that $A_{2}$ for sufficiently long chains vanishes when $\beta_{\mathrm{e}}=0$. Thus, $\Theta_{\infty}$, the theta point for infinite $n$, is the temperature at which a negative $\beta_{2}$ is compensated by a positive $C \beta_{3}$. As the molecular weight decreases, the second term in eq 7 may become significant. If the $\beta_{3}$ value of $(7 \pm 3) \times 10^{-46}$ $\mathrm{cm}^{6}$ estimated above is used together with $b=0.58 \mathrm{~nm}, A_{2}$ at $\Theta_{\infty}$ is found to fall below $-5 \times 10^{-6} \mathrm{~mol} \mathrm{~g}^{-2} \mathrm{~cm}^{3}$ at an $M$ of the order of $10^{6}$ where effects of chain ends ${ }^{22}$ are insignificant. This behavior of theoretical $\mathrm{A}_{2}$ disagrees with our experimental finding that $\left|A_{2}\right|$ for PIB in IAIV at $27^{\circ} \mathrm{C}$ is less than $5 \times 10^{-6} \mathrm{~mol} \mathrm{~g}^{-2} \mathrm{~cm}^{3}$ (i.e., virtually zero) down to $M_{w}=8 \times 10^{4}$. Thus, the current theories of the polymer virial coefficients fail to give a consistent explanation of the measured $\mathrm{A}_{2}$ and $A_{3}$ at $\Theta$. This is not specific to the present polymer + solvent system, since a similar inconsistency between theory and experiment was observed for polystyrene in cyclohexane. ${ }^{1}$ In sum, the molecular interpretation of the theta condition in terms of segment interactions still leaves much to be desired. It is one of the most basic problems in polymer physics.

\section{CONCLUSIONS}

The third virial coefficient for polyisobutylene in isoamyl isovalerate remains positive at the theta temperature and increases with 
decreasing temperature below $\Theta$. These features are very similar to what was found previously for polystyrene in cyclohexane, demonstrating that the binary cluster approximation to $A_{3}$ near $\Theta$ breaks down for these two typical flexible polymers in the theta solvents. Another important conclusion is that the current theories of the polymer virial coefficients are incapable of explaining the theta condition in terms of segment interactions.

\section{REFERENCES}

1. Y. Nakamura, T. Norisuye, and A. Teramoto, Macromolecules, 24, 4904 (1991).

2. H. Yamakawa, "Modern Theory of Polymer Solutions," Harper \& Raw, New York, 1971.

3. T. Matsumoto, N. Nishioka, and H. Fujita, J. Polym. Sci., A-2, 10, 23 (1974).

4. F. Abe, Y. Einaga, and H. Yamakawa, Macromolecules, 24, 4423 (1991).

5. Y. Nakamura, K. Akasaka, K. Katayama, T. Norisuye, and A. Teramoto, Macromolecules, 25, 1134 (1992).
6. Gj. Deželić and J. Vavra, Croat. Chem. Acta, 38, 35 (1966).

7. D. N. Rubingh and H. Yu, Macromolecules, 9, 681 (1976).

8. G. C. Berry, J. Chem. Phys., 44, 4550 (1966).

9. T. Sato, T. Norisuye, and H. Fujita, J. Polym. Sci., B: Polym. Phys., 25, 1 (1987).

10. Y. Nakamura, T. Norisuye, and A. Teramoto, $J$. Polym. Sci., B: Polym. Phys., 29, 153 (1991).

11. C. E. H. Bawn, R. F. J. Freeman, and A. R. Kamaliddin, Trans. Faraday Soc., 46, 862 (1950).

12. T. Norisuye and H. Fujita, ChemTracts-Macromol. Chem., 2, 293 (1991).

13. H. Murakami, T. Norisuye, and H. Fujita, Polym. J., 7, 248 (1975).

14. P. J. Flory and H. Daoust, J. Polym. Sci., 25, 429 (1957).

15. T. Konishi, T. Yoshizaki, and H. Yamakawa, Macromolecules, 24, 5614 (1991).

16. B. H. Zimm, J. Chem. Phys., 14, 164 (1946).

17. B. J. Cherayil, J. F. Douglas, and K. F. Freed, J. Chem. Phys., 83, 5293 (1985).

18. H. Yamakawa, J. Chem. Phys., 45, 2606 (1966).

19. O. Kratky and G. Porod, Rec. Trav. Chim., 68, 1106 (1949).

20. H. Yamakawa and W. H. Stockmayer, J. Chem. Phys., 57, 2843 (1972).

21. H. Yamakawa, Macromolecules, 26, 5061 (1993).

22. H. Yamakawa, Macromolecules, 25, 1912 (1992). 